\title{
Neuronal $A \beta 42$ is enriched in small vesicles at the presynaptic side of synapses
}

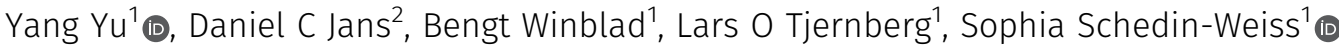

\begin{abstract}
The amyloid $\beta$-peptide (AB) is a physiological ubiquitously expressed peptide suggested to be involved in synaptic function, long-term potentiation, and memory function. The 42 amino acid-long variant $(A \beta 42)$ forms neurotoxic oligomers and amyloid plaques and plays a key role in the loss of synapses and other pathogenic events of Alzheimer disease. Still, the exact localization of $A \beta 42$ in neurons and at synapses has not been reported. Here, we used super-resolution microscopy and show that $A \beta 42$ was present in small vesicles in presynaptic compartments, but not in postsynaptic compartments, in the neurites of hippocampal neurons. Some of these vesicles appeared to lack synaptophysin, indicating that they differ from the synaptic vesicles responsible for neurotransmitter release. The A 342 -containing vesicles existed in presynapses connected to stubby spines and mushroom spines, and were also present in immature presynapses. These vesicles were scarce in other parts of the neurites, where $A \beta 42$ was instead present in large, around $200-600 \mathrm{~nm}$, vesicular structures. Three-dimensional super-resolution microscopy confirmed that $A \beta 42$ was present in the presynapse and absent in the postsynapse.
\end{abstract}

DOI 10.26508/lsa.201800028 | Received 5 February 2018 | Revised 1 June 2018 | Accepted 1 June 2018 | Published online 14 June 2018

\section{Introduction}

Several lines of evidence point towards amyloid $\beta$-peptide $(A \beta)$ being a causative agent in Alzheimer disease (AD), but the molecular details behind its role in the initiating events leading to clinical AD are elusive. Pathologically, the AD brain is characterized by extracellular plaques composed of fibrillary $A \beta$ surrounded by activated astrocytes, microglia, and dystrophic neurites. Other pathological hallmarks are accumulation of $A \beta$ in the vasculature, neurofibrillary tangles composed of hyperphosphorylated tau protein, brain atrophy, and loss of neurons and synapses (1). The accumulation of $A B$ in $A D$ brain starts several years to decades before clinical symptoms appear (2) and research during the past decades suggest that soluble forms of $A \beta$ rather than $A \beta$ plaques are neurotoxic and correlate with cognitive decline in $A D(3)$.
Different mechanisms behind $A \beta$ toxicity have been suggested. Several studies have shown that oligomeric forms of $A \beta$ can bind to cell surface receptors at neuronal synapses $(4,5)$, whereas other studies suggest that intracellular $A \beta 42$ is neurotoxic. Analyses of intraneuronal $A \beta$ levels in neurons dissected by laser capture microscopy from postmortem control and $A D$ brain showed that pyramidal neurons in cornu ammonis 1 of $A D$ hippocampus display increased $A \beta 42$ levels and increased $A \beta 42 / A \beta 40$ ratio in sporadic and familial AD cases (6). The study suggested that there is a correlation between high intracellular $A \beta 42$ levels and vulnerability to AD neuropathology. An electron microscopy study showed that the intracellular accumulation of $A \beta 42$ in human $A D$ brain and a transgenic AD mouse model (Tg2576) occurs predominantly within neuronal processes and an accumulation in synaptic compartments was observed (7). The neurotoxicity of intracellular $A \beta$ is also supported by a study in which autophagy-deficient mice were cross-bred with amyloid precursor protein (APP) transgenic mice (8). Intriguingly, the cross-bred mice show impaired secretion of $A \beta$, resulting in highly increased intracellular levels of $A \beta$. This increase was accompanied by severe memory impairment, significantly worse compared with the APP transgenic mice (8).

An increasing amount of studies suggest that $A \beta$ is of importance in normal synaptic function, such as developmental synaptic plasticity (9). Thus, it is important to study the synthesis, localization, and function of $A \beta$ both from a physiological and pathological perspective. A $\beta$ is formed from the APP by the sequential action of two transmembrane aspartyl proteases. BACE-1 first cleaves APP to generate a 99-residue C-terminal fragment (C99), which is subsequently cleaved by the protein complex $y$-secretase, generating an APP intracellular domain (AICD) and A of variable lengths $(10,11)$. The most common form contains 40 amino acids (A 40$)$, whereas the longer forms $(A \beta 42$ and $A \beta 43)$ are more aggregation-prone and neurotoxic $(12,13)$. Several studies have shown that APP can be transported along axons and dendrites to nerve terminals $(14,15,16)$. Moreover, BACE1 and $\gamma$-secretase have been found to be enriched at the neuronal synapse and it has been suggested that $A \beta$ is indeed produced at the synapse $(17,18)$. Whether or not neuronal $A \beta$ is secreted in an activity-dependent manner has been debated, and a recent study suggested that the

${ }^{1}$ Center for Alzheimer Research, Division of Neurogeriatrics, Department of Neurobiology, Care Sciences, and Society, Karolinska Institutet, Huddinge, Sweden ${ }^{2}$ Science for Life Laboratory, Department of Applied Physics, Royal Institute of Technology, Stockholm, Sweden 
release of $A \beta$ is mostly constituent and not significantly correlated to the activity-dependent release of glutamate (19). However, neither the physiological importance nor the mechanism by which this occurs has been clarified.

Here, we asked the following questions: Is $A \beta 42$ found all over neurites or enriched at the synapse? Is it found on the pre- or postsynaptic side or both? Which intracellular structures/organelles is it associated with? Confocal microscopy with a best possible resolution of around $200 \mathrm{~nm}$ is not sufficient to resolve most synaptic structures. For instance, synaptic vesicles are $40-50 \mathrm{~nm}$ in diameter and the synaptic cleft is only 20-25 nm wide. Therefore, we used superresolution microscopy to study the localization of endogenously derived $A \beta 42$ in the neurites of primary hippocampal neurons. Intriguingly, we were able to resolve different types of fine structures of intracellular A 42 that cannot be resolved by confocal microscopy, revealing novel information about the synaptic localization of A 42 that may be crucial for understanding its physiological function and the mechanism behind its neurotoxicity.

\section{Results and Discussion}

This study was performed to determine the precise localization of endogenous $A \beta 42$ in neurites and synapses in cultured hippocampal neurons by super-resolution microscopy. It has previously been shown that intraneuronal levels of $A \beta 42$ correlate with neuropathology in $\operatorname{AD}(20,21)$. There is also a correlation between synaptic degeneration and development of $A D$, and $A \beta 42$ has been shown to be synaptotoxic $(4,16,22,23)$. Because the synaptic cleft is only 20-25 $\mathrm{nm}$ wide (24) and the best possible resolution obtained by confocal microscopy is around $200 \mathrm{~nm}$, super-resolution microscopy is required to determine the synaptic localization of macromolecules with sufficient resolution and precision. A recent study using super-resolution microscopy to investigate the localization of the $A \beta$-producing protease $y$-secretase in the neurites showed that this enzyme is enriched at the synapse and that it is present at both the pre- and the postsynaptic side (18). Because $y$-secretase can process around 100 substrates besides APP, it is important to determine at which subcellular localizations it can perform its various activities. Here, we studied the localization of A 42 in the neurites by using an A 42 -specific antibody. This antibody has been thoroughly characterized previously $(25,26)$. It was further validated here by Western blotting (WB) (Fig S1), supporting the notion that this $A \beta 42$-specific antibody targets the free $\mathrm{C}$-terminus of $A \beta 42$ and has no cross-reactivity with $A \beta 40$ or full-length APP. The presynaptic marker synaptophysin and the postsynaptic marker PSD95 were visualized by antibodies previously shown to be suitable for stimulated emission depletion (STED) and stochastic optical reconstruction microscopy (STORM) imaging of hippocampal neurons (18). The structure of the neurites was visualized using phalloidin, which binds to filamentous actin. STED experiments were set up with two STED channels, one for A 42 and one for either of the two synaptic markers (synaptophysin or PSD95). The same depletion doughnut was used in both STED channels to ensure perfect colocalization between these two channels. The actin pattern was imaged in a third, confocal channel throughout this study. The synaptic localization of A 42 was further confirmed by two-color dSTORM experiments.

The fluorescence labeling of Aß42, synaptic markers, and filamentous actin was first optimized and analyzed by confocal microscopy. The culturing of mouse primary hippocampal neurons for $21 \mathrm{~d}$ in vitro results in an elaborate network of axons and dendrites with well-developed specialized synaptic contacts, including dense PSD clusters in the spine heads and enrichment of synaptophysincontaining synaptic vesicles on the opposing, presynaptic side $(18,27)$. The specificity of the fluorescence labeling was analyzed by using negative controls, lacking one of the primary antibodies, to confirm that there was no cross-talk between the channels and that the secondary antibodies did not cross-react (Fig S2). In agreement with previous studies, A $\beta 42$ staining was present in the perinuclear region and in the neurites (27).

\section{STED resolves structures and localization of $A \beta 42$ that cannot be resolved by confocal microscopy}

Confocal images of the network of the neurites in samples stained for A 42, synaptophysin, and filamentous actin showed an accumulation of $A \beta 42$ at the synapse, but it was not possible to distinguish whether $A \beta 42$ was localized to the pre- or postsynapses (Fig 1A). Imaging the same regions using STED microscopy we were able resolve the fine structures in the neurites (Fig 1B). Close-ups of the STED images (Fig 1B, middle and right panels) demonstrated that synaptophysin and $A \beta 42$ are interspersed within the same regions in the neurites, suggesting that $A \beta 42$ is present at the presynapse. Because our previous study showed that axonal $\gamma$-secretase is enriched in the presynapse (18), we propose that this pool of intracellular endogenous $A \beta 42$ is synthesized in the presynaptic region.

A comparison between confocal and STED microscopy was also performed for samples stained for the postsynaptic marker PSD95, $A \beta 42$, and filamentous actin (Fig $1 C$ and D). Confocal microscopy showed that AB42 clusters were located close to PSD95-containing spine heads (Fig 1C). With STED microscopy we were able to reveal that such clusters contain small vesicles adjacent to, but not colocalizing with, the postsynaptic spine heads (Fig 1D), in line with a presynaptic localization. At a nonsynaptic part of a neurite, we noted a different type of A 42 structure, which only contained $A \beta 42$ on the rim and was much larger than the vesicles found at the synapse (Fig 1D and see below for more details). Notably, these different A 42 -containing structures, small vesicles at the synapse and larger nonsynaptic bodies, could only be resolved and differentiated by using STED. Our findings thus demonstrate that super-resolution microscopy is required both to determine the synaptic localization of $A \beta 42$ and to distinguish between different types of $A \beta 42$ structures in the neuronal compartments.

Although the actin staining is present both in dendrites and in most of the axons, the staining in dendrites is considerably denser, particularly at the spine heads, whereas the axonal staining is weaker (18). However, by adapting contrast settings in the images, it is possible to optimize visualization either of the densely stained dendrites (Fig 2A and B, left panels) or, with increased brightness, the axons (Fig 2A and B, right panels). As in previous studies, the 


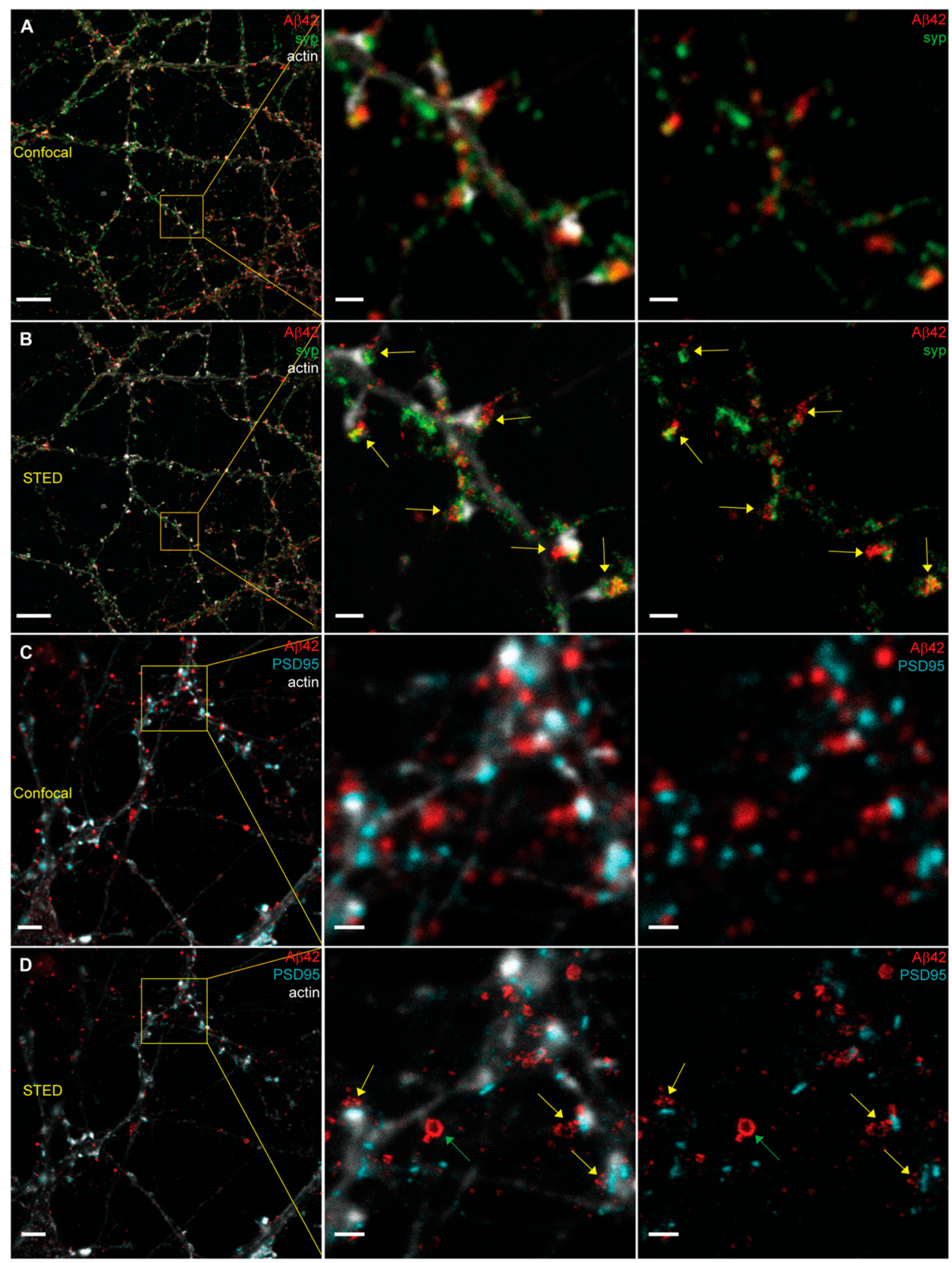

Figure 1. Comparison of confocal and STED images for studying the synaptic localization of A $A 42$ in hippocampal neurons.

(A, B) Hippocampal neurons stained to visualize Aß42 (red), synaptophysin (green), and actin (light grey) were imaged by confocal microscopy (A) and STED microscopy (B). Yellow arrows point at enrichment of A 42 at the synapse. Left scale bars: $10 \mu \mathrm{m}$. Other scale bars: $1 \mu \mathrm{m}$. (C, D) Hippocampal neurons subjected to immunocytochemistry to visualize AB42 (red), PSD95 (cyan), and actin (light grey) were imaged by confocal microscopy (C) and STED microscopy (D). Yellow arrows point at enrichment of $A \beta 42$ at the synapse. Green arrow points at an Aß42-stained vesicle where A 42 is stained only at the rim of the vesicle. Left scale bars: $4 \mu \mathrm{m}$. Other scale bars: $1 \mu \mathrm{m}$. 

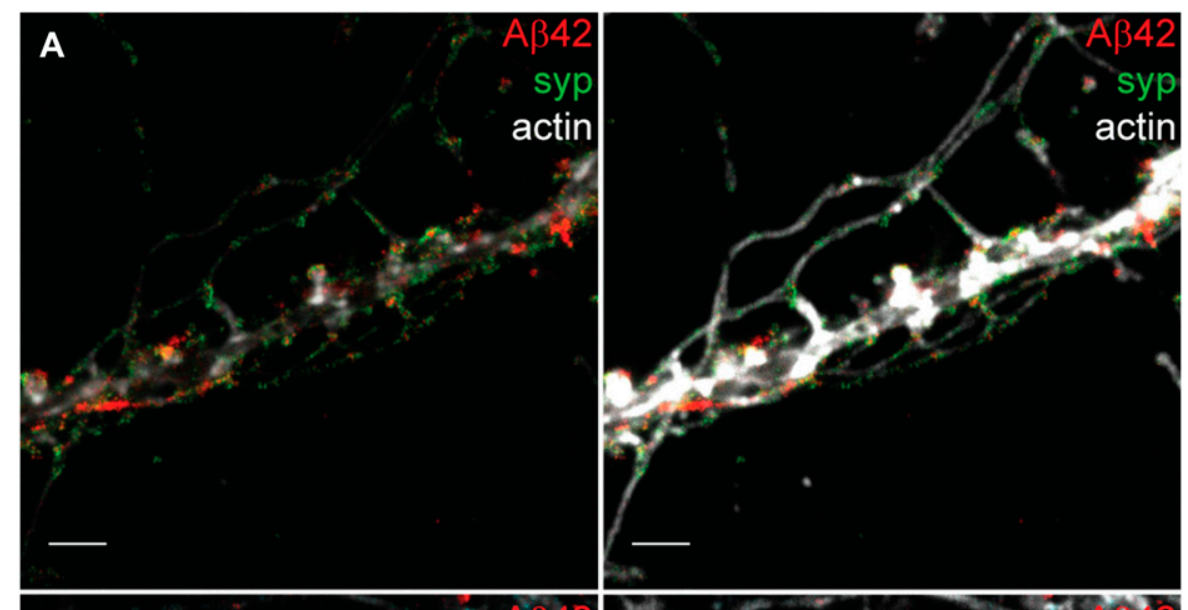

Figure 2. STED images of $A \beta 42$ demonstrating the intricate axonal network surrounding dendrites in the 21-d in vitro hippocampal neurons.

(A) STED image showing A 42 (red) and synaptophysin (green) combined with a confocal channel for actin (light grey). The left image has a lower brightness in the actin channel than the right image. Note that the axonal staining appears for many axons only in the right image with increased brightness. This image was further zoomed-in in Fig 3A to focus on the synapses. Scale bars: $2 \mu \mathrm{m}$. (B) STED image showing Aß42 (red) and PSD95 (cyan) combined with a confocal channel for actin (light grey). The left image has a lower brightness in the actin channel than in the right channel. Note the intensive axon network both between and along the neurites. Scale bars: $2 \mu \mathrm{m}$.

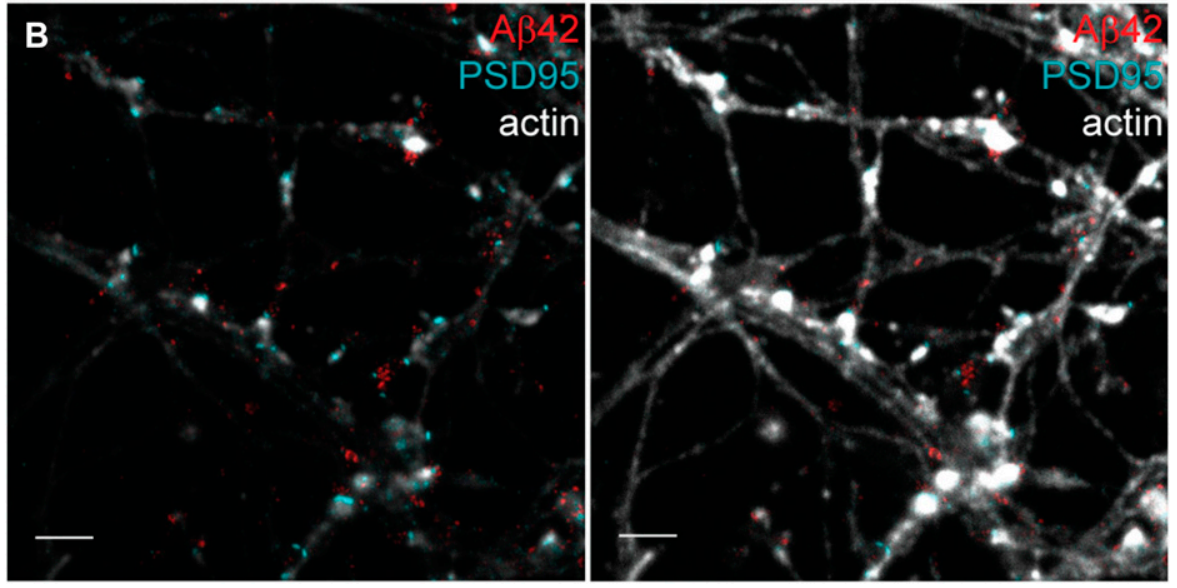

dendrites have extensive axonal networks along with as well as between them (18).

\section{Analysis of the distribution of $A \beta 42$ at the pre- and postsynaptic sides by STED}

Analyzing a large number of synapses from different areas of the dish, we found that most of the AB42 staining in the neurites was present within the same region as the synaptophysin clusters. Interestingly, both A 42 and synaptophysin appeared to be present in vesicular structures of similar size, and to some extent colocalized (Figs $3 \mathrm{~A}$ and $\mathrm{B}$, and $\mathrm{S} 3 \mathrm{~A}$ and $\mathrm{B}$ ). The appearance of the synaptophysin staining is in agreement with previous electron microscopy studies showing that synaptic vesicles are uniform in size with a diameter of around 40-50 nm (28). It is highly intriguing that we now observed that some vesicles of similar size as synaptic vesicles stained for $A \beta 42$ but not synaptophysin. Because colocalization of $A \beta 42$ and synaptophysin was observed in some areas, it is unlikely that the absence of synaptophysin staining in these vesicles is due to steric hindrance of the antibody but rather indicates the presence of A 42 -containing vesicles that lack synaptophysin. To further test this hypothesis, we compared the samples stained for A 42 and synaptophysin with samples stained for synaptophysin and another synaptic vesicle protein-synaptobrevin. In the presynaptic regions of the A 342 /synaptophysin samples, $19 \%$ of the stained pixels were positive for only $A \beta 42,36 \%$ were positive for both $A \beta 42$ and synaptophysin, and $45 \%$ were positive for only synaptophysin (Fig S4). Thus, 35\% of the AB42-stained pixels did not colocalize with synaptophysin and $56 \%$ of the synaptophysin-stained pixels did not colocalize with A 42 (Fig S4). Doing the same operation for the synaptobrevin/synaptophysin samples, we found that $15 \%$ of the stained pixels were positive for only synaptobrevin, 52\% were positive for both synaptobrevin and synaptophysin, and 33\% were positive for only synaptophysin. Thus, only $22 \%$ of the synaptobrevinpositive pixels did not colocalize with synaptophysin, and $39 \%$ of the synaptophysin-positive pixels did not colocalize with synaptobrevin. The synaptobrevin/synaptophysin colocalization was somewhat lower than we expected, possibly because of labeling efficacies lower than $100 \%$. Another potential explanation is that the size of the primarysecondary antibody complex may provide a detection area somewhat larger than the actual vesicle size. Alternatively, the vesicle pool could be more heterogeneous than expected. Still, the lower extent of colocalization of A 342 /synaptophysin compared with synaptobrevin/ synaptophysin in the presynaptic region supports our notion that part of the AB42-containing vesicles may be distinct from the synaptic vesicles responsible for neurotransmitter release. Previous work has shown that $A \beta 42$ release can occur independently of glutamate release (19), supporting our notion that a fraction of $A \beta 42$ is present in vesicles that differ from the vesicles responsible for transmitter release. 
踽 Life Science Alliance
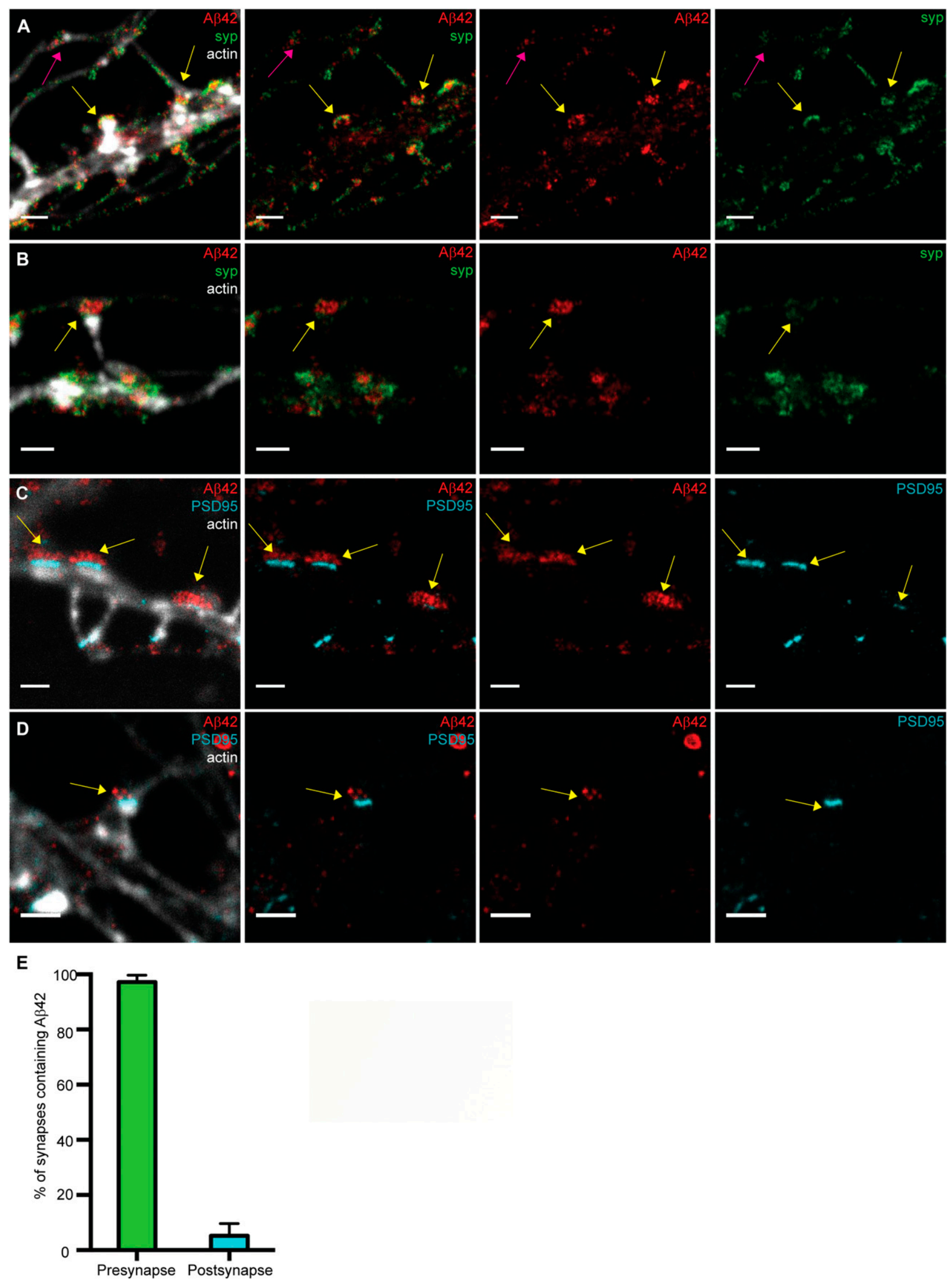
The appearance of vesicles that contain A 42 and/or synaptophysin was observed both in immature presynapses that have not yet formed a contact with a dendritic spine (Fig 3A) and in presynapses that are in contact with dendritic spine heads (Fig 3B). Notably, our previous super-resolution microscopy study showed that the enzyme complex $\gamma$-secretase, which is required for the generation of $A \beta 42$, is also present in both immature and mature synapses (18). Together, these findings imply that $A \beta 42$ and/or $y$-secretase have a role(s) in synaptic formation and maintenance.

PSD95 imaging by STED beautifully outlined PSD clusters at the top of the spine heads and these clusters, in contrast to synaptophysin clusters, showed no or only little overlap with A 42 (Figs $3 C$ and D, and S3C and D). A clear enrichment close to the PSD clusters was observed, on the other side of the synapse, again supporting the presynaptic localization of A 42 . This presynaptic clustering of A 42 vesicles opposite to PSD clusters was obvious both for stubby spines (Fig 3C) and mushroom spines (Fig 3C and D). Quantification of individual synapses stained for $A \beta 42$, phalloidin, and synaptophysin or PSD95 showed that the majority $(97 \pm 2.5 \%)$ of presynapses were positive for $A \beta 42$ vesicles, whereas only very few of the postsynapses $(5.2 \pm 4.5 \%)$ were positive for A 42 staining (Fig 3E). The latter could be because of a small extent of false positive results if some of the dendritic spines were not perfectly aligned perpendicular to the optical axis, i.e., because of the lower resolution along the z-axis.

\section{A 342 is present in large vesicles in nonsynaptic regions of the neurites}

The occurrence of larger A 42 -containing structures (Fig 1D), with a diameter ranging between 200 and $600 \mathrm{~nm}$ (Fig S5) and enriched in $A \beta 42$ in the outer portions, were further investigated. Although the staining was intense at the rim, $A \beta 42$ is most likely enriched in the interior of the vesicle, close to the membrane. In contrast to the smaller vesicles, these vesicles were located on or close to neurites in regions lacking spine heads, synaptophysin, or PSD95 clusters (Fig 4A and B), indicating that they are not present at the synapse. Interestingly, a previous electron microscopy study showed that $A \beta 42$ is enriched at the membrane of multivesicular bodies (MVBs) in neurons both in human AD brain and in brain from mice and rats, especially in pyramidal neurons from the cortex and hippocampus, which are most prone to develop AD neuropathology (7). Furthermore, it has also been shown that $A \beta$ can be produced in a macroautophagy-dependent pathway involving autophagosomes and late autophagic vacuoles, which are multivesicular entities with a diameter of around $500 \mathrm{~nm}$ (29). Because intraneuronal A 42 can be directly derived by endogenous production or secreted and taken up by endocytosis of external $A \beta 42$, it is possible that the sources of $A \beta 42$ in MVBs and presynaptic vesicles are of different origin.

\section{Analysis of the distribution of $A \beta 42$ at the pre- and postsynaptic sides by dSTORM}

The size of synaptic structures and vesicles makes diffraction unlimited super-resolution microscopy indispensable for investigating synaptic localizations of proteins. All diffraction unlimited techniques rely on switching between two discernible states, and two classes of diffraction unlimited super-resolution techniques can be distinguished: spatially targeted methods such as STED and spatially stochastic techniques such as, for instance, photoactivated localization microscopy and STORM (30, 31). All these methods can overcome the diffraction barrier substantially. So, we asked if the synaptic localization of A 42 can also be seen using a spatially stochastic super-resolution technique. Here, we applied dSTORM $(32,33)$, using the two photo-switchable fluorophores, Alexa Fluor 647 and Atto 488, as reporters for A 42 and the synaptic marker, respectively. In agreement with the STED images, $A \beta 42$ was dispersed within the same region as synaptophysin (Fig 5A) but not as PSD95 (Fig 5B), supporting our notion that $A \beta 42$ is localized at the presynapse in pyramidal neurons. Importantly, there was no or very little direct overlap between $A \beta 42$ and synaptophysin staining. These findings show that A 42 localization can be analyzed also by using spatially stochastic approaches and support our suggestion that $A \beta 42$ is partially present in vesicles different from those containing synaptophysin.

\section{D STED confirms the presynaptic localization of $A \beta 42$}

The confocality of a STED microscope and the easy implementation of additional (confocal) reference channels make it particularly suitable also for analyzing complex structures such as neuronal cultures. To analyze pre- or postsynaptic protein localizations, the positioning of the synapse with respect to the optical axis of the microscope has to be considered. Conventional 2D STED using a vortex phase plate provides very high lateral resolution but the axial resolution is still unaltered confocal, which means that structures that are aligned along this axis cannot be resolved beyond the diffraction limit. Therefore, we focused in the previous part on synapses that are aligned perpendicular to the optical axis. To analyze the localization of A 42 in a 3D context, we used 3D STED, providing super-resolution in all directions. The presynaptic localization of A 342 could be confirmed using 3D STED. We found A 42 partially colocalizing with synaptophysin (Video 1), whereas the lack of overlap between the PSD95 and Aß42 staining (Video 2) was apparent in all three dimensions, confirming our 2D STED observations.

In summary, by using super-resolution microscopy we could, in hippocampal neurons, show that endogenous $A \beta 42$ is present at

Figure 3. STED imaging of $A \beta 42$ focusing on the pre- or postsynaptic regions.

(A, B) Zoomed STED images of synapses showing Aß42 (red) and the presynaptic marker synaptophysin (green). Neuronal structure is shown by actin in a confocal channel (light grey) merged to the STED channels in the left panel. The staining of synaptophysin and Aß42 at presynaptic boutons connected to the postsynaptic spines are shown by the yellow arrows. The staining of synaptophysin and Aß42 at "free" boutons that do not connect to the postsynaptic spine is shown by a pink arrow. Scale bars: $1 \mu \mathrm{m}$. (C, D) Zoomed STED images of synapses showing A 42 (red) and the postsynaptic marker PSD95 (cyan). Neuronal structure is shown by actin staining in a confocal channel (light grey) merged to the STED channels in the left panel. Yellow arrows point at Aß42 vesicles in axonal regions opposite to PSD95-containing stubby spines in (A) and to A 42 vesicles in axonal regions opposite to a mushroom spine in (B). Scale bars: $1 \mu \mathrm{m}$. (E) Quantification of Aß42 staining in pre- and postsynapses. The staining of A 42 combined with the presynaptic marker synaptophysin (green bar) or postsynaptic marker PSD95 (cyan bar) was quantified from STED data. Graphpad prism was used to prepare graphs showing the proportion \pm SD of presynapses $(n=73)$ and postsynapses $(n=73)$ that were positively stained by A 42 . 


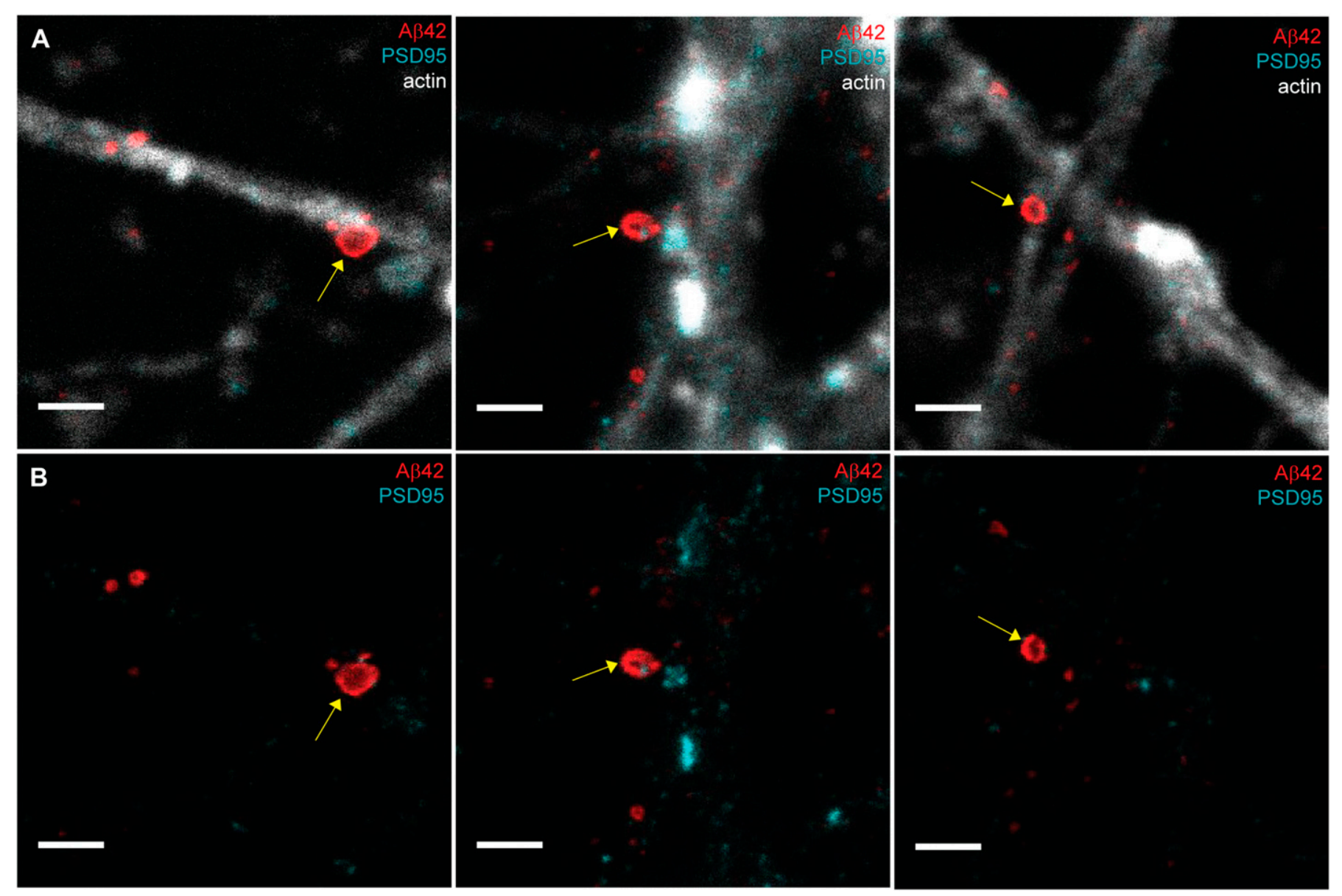

Figure 4. A $A$ 42 staining at nonsynaptic neuritic regions.

Zoomed STED images of AB42 (red), PSD95 (cyan), and actin (light grey) at nonsynaptic neuritic regions (A) and only the Aß42 and PSD95 channel (B). Yellow arrows show A 42 vesicles which are hollow inside. The diameter from left to right is 520, 480, and $490 \mathrm{~nm}$, respectively. Images were zoomed from Fig S3. Scale bars: 1 Hm.

the pre- but not postsynaptic side. Interestingly, presynaptic Aß42 was localized to vesicles of similar size as synaptic vesicles, some of which lacked synapthophysin, suggesting that a fraction of $A \beta 42$ is present in a previously non-characterized vesicle in the presynapse. Intriguingly, these data may explain the controversy regarding the dependency of $A \beta$ secretion on synaptic activity. Moreover, we could also resolve larger, nonsynaptic structures in which $A \beta 42$ showed a donut-like appearance, in line with the presence of $A \beta 42$ in the outer membrane of MVB. Thus, these findings provide crucial information on the subcellular details and the molecular mechanisms behind the role of $A \beta 42$ in normal synaptic function and in AD pathogenesis.

\section{Materials and Methods}

\section{Mice}

The C57BL6 mice used in this study for preparing hippocampal neurons and mouse brain homogenate were treated according to the Karolinska Institutet's and the national guidelines. Embryonic mice brains (E16.5) were used for the dissection of hippocampi. The brains of the female mice carrying the embryos were used for WB. After dissection, these brains were homogenized in a buffer containing $20 \mathrm{mM}$ Hepes, $50 \mathrm{mM} \mathrm{KCl}, 2 \mathrm{mM}$ EGTA, and complete protease inhibitor cocktail (Roche), at pH 7.5. No experiments were performed on live animals. The study was approved by the animal research ethical committee in southern Stockholm. Animals were euthanized by cervical dislocation.

\section{Hippocampal cell culture}

Mouse hippocampal neurons were cultured in 35- $\mathrm{mm}$ glass bottom culture dishes (P35-G-1.5-10-C; MatTek) with 2\% B27 (Thermo Fisher Scientific) and 1\% L-glutamine (Gibco) in Neurobasal medium (Gibco), essentially as described previously $(34,35)$. Briefly, the dishes were coated with poly-D-lysine (P7405; Sigma) at least $1 \mathrm{~d}$ before the dissection. Hippocampi and cortices were dissected and single cells were triturated by repeated pipetting of the tissue in cell medium. 7,500 hippocampal cells (with $125 \mu$ l medium) were seeded on glass in the middle of the plate, and 150,000 cortical cells (with $500 \mu \mathrm{l}$ medium) were seeded as support cells on the surrounding plastic (to avoid direct contact between the cell types). The cells were kept in the incubator $\left(37^{\circ} \mathrm{C}, 5 \% \mathrm{CO}_{2}\right)$ for $2 \mathrm{~h}$ and then 

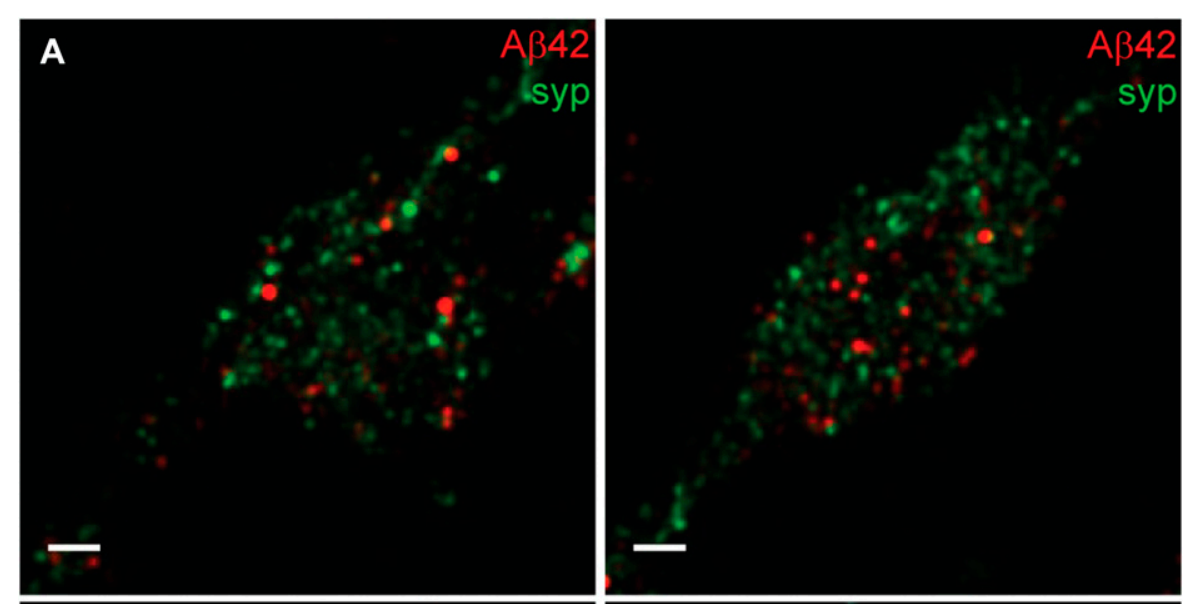

Figure 5. Two-color dSTORM images of A $\beta 42$ and pre- or postsynaptic markers.

(A) dSTORM images show A 42 (red) and

synaptophysin (green). Two different presynaptic regions from the same image are shown in the left and right image. Note that the $A \beta 42$ staining appears as vesicles that are interspersed in the same region as synaptophysin. Scale bars: $500 \mathrm{~nm}$. (B) STORM images show AB42 (red) and PSD95 (cyan). Two different postsynaptic regions from the same image are shown in the left and right image. Note the proximity between A 42 and PSD95, in agreement with a synaptic cleft between the two stains. The somewhat overlap in the left panel is probably related to the fact that the synapse is not in the plane of the image. Scale bars: $500 \mathrm{~nm}$.
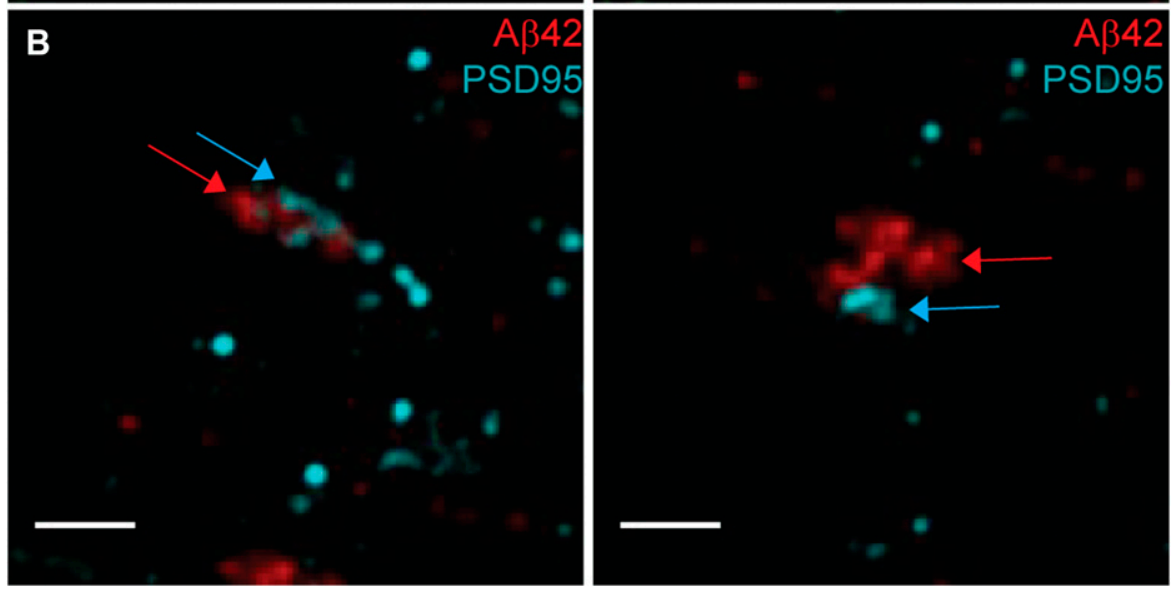

$2.5 \mathrm{ml}$ fresh medium was added. After $21 \mathrm{~d}$, the cells were fixed with $4 \%$ formaldehyde (252549; Sigma) for $10 \mathrm{~min}$ at RT and stored in Dulbecco's phosphate-buffered saline (DPBS) (Gibco) at $4^{\circ} \mathrm{C}$ for immunocytochemistry staining.

\section{Antibodies and reagents}

A rabbit antibody specific for the C-terminal neo-epitope of $A \beta 42$ (anti-ABC42) was used for immunofluorescence labeling of $A \beta 42$ throughout these studies. This affinity-purified antibody, targeting the six C-terminal residues of $A \beta 42$, has previously been shown not to cross-react with either A 40 or full-length APP (25). To further validate the antibody, WB blotting was performed and compared with mouse antibody $4 \mathrm{G} 8$ (Biolegend), which is reactive to amino acid residues 17-24 of the A sequence within APP. IRDye 800CW (LI-COR) and IRDye 680RD secondary antibodies (LI-COR) were used for WB.

Mouse anti-PSD95 IgG, here denoted anti-PSD95 (Ab2723; Abcam), was used as a postsynaptic marker whereas mouse antisynaptophysin IgG, here denoted anti-synaptophysin (SP15; Enzo Life Sciences), was used as a presynaptic marker. Mouse antisynaptobrevin IgG, here denoted anti-synaptobrevin (104203; Synaptic Systems), was used as another presynaptic marker. All of these antibodies have previously been shown to be suitable for confocal, STED, and STORM experiments (18). The Atto 488-labeled secondary antibody used for dSTORM was prepared by the conjugation of
NHS-Atto 488 (ATTO-TEC) to donkey anti-mouse IgG (A16019; Life Technologies). Labeling was performed essentially as described previously (18) by incubating Atto 488-NHS (0.02 mg/tube) dissolved in $10 \mu \mathrm{l}$ anhydrous DMSO, with $50 \mu \mathrm{l}$ donkey anti-mouse IgG $(1.25 \mathrm{mg} / \mathrm{ml}$ in PBS), $6 \mu \mathrm{l}$ of $1 \mathrm{M} \mathrm{NaHCO}_{3}$, and $1.5 \mu \mathrm{l}$ Atto 488-NHS for for $30 \mathrm{~min}$ at RT, followed by purification using size exclusion chromatography on NAP-5 columns (GE Healthcare). Fluorescently labeled commercial secondary antibodies used were anti-rabbit IgG Fab Alexa Fluor 647 (Invitrogen), anti-rabbit IgG Abberrior STAR 635P, and anti-mouse IgG Alexa Flour 594 (Invitrogen). The neuronal structure was visualized by staining the actin cytoskeleton with Alexa Flour 488-phalloidin (Invitrogen).

The imaging buffer for STORM experiments containing $0.124 \mathrm{M}$ cysteamine (Sigma), $44.8 \mathrm{mM} \mathrm{HCl}, 8.6 \%$ glucose, $1.08 \mathrm{mg} / \mathrm{ml}$ glucose oxidase from Aspergillus niger (Sigma), and $0.0773 \mathrm{mg} / \mathrm{ml}$ catalase from bovine liver (Sigma) in PBS was mixed directly before imaging and used for no longer than $1 \mathrm{~h}$. Fresh stock solutions (1 M cysteamine in $360 \mathrm{mM} \mathrm{HCl}, 10 \%$ Glucose in PBS, $70 \mathrm{mg} / \mathrm{ml}$ glucose oxidase in PBS, and $20 \mathrm{mg} / \mathrm{ml}$ catalase in PBS) were prepared the day before imaging and stored at $4^{\circ} \mathrm{C}$.

\section{WB}

Purified recombinant human $A \beta 42$ and mouse brain homogenate samples were diluted with LI-COR sample loading buffer and 
separated on 4\%-12\% Bis-Tris gel (Invitrogen) in MES SDS running buffer (Invitrogen). Proteins were transferred onto nitrocellulose membranes (GE Healthcare). Membranes were blocked by Odyssey blocking buffer (927-50000; LI-COR) for $1 \mathrm{~h}$ at RT, followed by incubation with A 42 -specific antibody and $4 \mathrm{G} 8$ primary antibody overnight. The membranes were washed with $0.1 \%$ Tween-TBS for $3 \times$ 5 min, incubated with anti-mouse and anti-rabbit LI-COR secondary antibodies for $1 \mathrm{~h}$ at RT, washed again, and followed by imaging in the Odyssey imaging system. The system can detect two protein targets that have been incubated with two different secondary antibodies labeled with spectrally distinct near-infrared fluorophores. The results can be shown as a merge image and as separate 700- and 800-nm channel images in pseudo-color or grayscale.

\section{Sample preparation for STED and dSTORM}

Fixed cells were permeabilized in $0.4 \%$ CHAPSO (Merck Millipore) in DPBS for 10 min at RT and blocked with 10\% normal goat serum (NGS; Invitrogen) for $15 \mathrm{~min}$ at RT. Dishes were subsequently incubated with the primary antibodies in 3\%-5\% NGS in DPBS overnight at $4^{\circ} \mathrm{C}$.

For the preparation of samples for STED imaging, anti-A $\beta 42$ (1:100) was used in combination with either anti-PSD95 diluted 1:200 or mouse anti-synaptophysin diluted 1:200 followed by washing with PBS $3 \times 5 \mathrm{~min}$. For colocalization studies, anti-synaptophysin (1:200) was also used in combination with anti-synaptobrevin (1:200). Incubation with the secondary antibodies, anti-rabbit Abberior Star 635P (1:200), anti-mouse Alexa Fluor 594 (1:200), and Alexa Fluor 488-phalloidin (1:100), was subsequently performed for 2-3 h, followed by washing with $0.1 \%$ tween in PBS for $3 \times 10$ min and PBS for $1 \times 5$ min. The samples were postfixed with $3 \%$ formalin and $0.1 \%$ glutaraldehyde in DPBS for 10 min at RT. After a final washing step for $3 \times 5$ min with DPBS and $1 \times 5$ min with water, the samples were mounted with ProLong gold antifade reagent (Life Technologies) and stored in the fridge for staining. The samples were first analyzed by using a Nikon point scanning confocal $\mathrm{A} 1+\mathrm{Si}$ inverted microscope using a 100 (NA: 1.55) oil immersion objective with an image size of 1,024 × 1,024 pixels. Excitation lasers were 405, 488, 561, and $640 \mathrm{~nm}$.

For the preparation of samples for dSTORM imaging, anti-A 42 (1:200) was used in combination with either anti-PSD95 diluted 1:200 or mouse anti-synaptophysin diluted 1:500.

After the incubation, the dishes were washed with PBS for $3 \times 5$ min. Subsequently, they were incubated with secondary antibodies, antirabbit IgG Fab 647 diluted 1:200 (Invitrogen) and anti-mouse IgG Atto 488 diluted $1: 200$, in $5 \%$ NGS in DPBS for $2-3 \mathrm{~h}$ followed by washing with $0.1 \%$ tween in PBS for $3 \times 5$ min and PBS for another $3 \times 5$ min. The samples were subsequently postfixed with $3 \%$ formalin and $0.1 \%$ glutaraldehyde (Sigma) in DPBS for 10 min at RT. The final washing step was performed for $3 \times 5$ min with DPBS and $1 \times 5$ min with water, followed by storage in DPBS until imaging.

\section{STED imaging}

STED imaging was performed on a Leica TCS SP8 STED $3 \times$ (Leica Microsystems) equipped with an HC PL APO 100×/1.40 Oil STED WHITE objective. In brief, the fluorophores Alexa Fluor 594 and
Abberior Star 635P were excited at 598 and $653 \mathrm{~nm}$, respectively, and STED was performed at $775 \mathrm{~nm}$ for both color channels. The dye Alexa Fluor 488 was excited at $499 \mathrm{~nm}$ and recorded confocally. The channels were recorded sequentially. All images except the movies are raw data. No image processing, except for contrast stretching, was applied. The 3D STED data were deconvolved using Huygens deconvolution software (Scientific Volume Imaging) and rendered and animated using Imaris (Bitplane).

\section{dSTORM imaging}

dSTORM imaging was performed using an Elyra PS.1 microscope (Carl Zeiss Microscopy) equipped with a Plan-Apochromat 100×/1.46 oil objective and a liquid cooled EMCCD camera (Andor Technology). Imaging was carried out in MEA imaging buffer as previously described (36). In short, fresh stock solutions (1 M cysteamine in $360 \mathrm{mM} \mathrm{HCl}, 10 \%$ glucose in PBS, $70 \mathrm{mg} / \mathrm{ml}$ glucose oxidase in PBS, and $20 \mathrm{mg} / \mathrm{ml}$ catalase in PBS) were prepared the day before imaging and stored at $4^{\circ} \mathrm{C}$ and mixed directly before imaging to final concentrations of $0.124 \mathrm{M}$ cysteamine (Sigma), $44.8 \mathrm{mM} \mathrm{HCl}$, $8.6 \%$ glucose, $1.08 \mathrm{mg} / \mathrm{ml}$ glucose oxidase from Aspergillus niger (Sigma), and $0.0773 \mathrm{mg} / \mathrm{ml}$ catalase from bovine liver (Sigma) in PBS. Imaging was performed in $12.8 \times 12.8-\mu \mathrm{m}$ areas in an inclined total internal reflection fluorescence microscope mode (37). Single molecule fluorescence detection on the EMCCD camera was acquired with $100 \times 100-n m$ pixel size, 20-ms Exposure time, and 100 Gain. 20,000 image frames were acquired for each channel. Both channels were imaged sequentially in 500 frame sequences and the appropriate filters and lasers for each dye were used $(642 \mathrm{~nm}$ for Alexa Fluor 647 and $488 \mathrm{~nm}$ for Atto 488). The images were analyzed with the ImageJ plugin SMLocalizer (38).

\section{Quantification of $A \beta 42$ in the synapse}

The relative presence of $A \beta 42$ in the pre-versus postsynaptic side of the synapse was quantified from STED images of A 42 , actin, and synaptophysin or PSD95 staining. The synapses to be included were selected based on the staining of actin and the synaptic markers (synaptophysin for the presynaptic side and PSD95 for the postsynaptic side). Criteria used for selecting synapses were as follows: (i) distinct spine structure (revealed by actin staining) in the plane of the slide; (ii) having a connection to a presynaptic bouton on the side; (iii) not overlapping with other dendrite or axon; and (iv) presence of a PSD cluster in the spine head (for postsynaptic localization) or synaptophysin cluster in a bouton (for presynaptic localization). The selected synapses were marked in Photoshop by yellow circles (Figs S6 and S7). A total of 73 pre- or postsynapses were selected from three to four different images. Graphs showing the proportion \pm SD of pre- and postsynapses with $A \beta 42$ staining were prepared using Graphpad prism.

\section{Comparison of colocalization of $A \beta 42 /$ synaptophysin with synaptobrevin/synaptophysin}

The STED images of A 42 /synaptophysin and synaptobrevin/ synaptobrevin were analyzed using ImageJ. Presynaptic regions were manually selected as regions of interest. The threshold was 
set by using the Otsu algorithm, and colocalization was analyzed based on the area of staining. A total of 50 presynapses were selected for each staining combination and the percentage of Aß42 or synaptobrevin colocalized with synaptophysin was displayed using Venn charts. Total stained pixels in each chart was set to 100\% (Fig S4).

\section{Supplementary Information}

Supplementary Information is available at https://doi.org/10.26508/lsa. 201800028.

\section{Acknowledgements}

We acknowledge the Advanced Light Microscopy facility at Science for Life Laboratory included in the National Microscopy Infrastructure (VR-RFI 201600968) for excellent support with super-resolution fluorescence imaging. L Tjernberg acknowledges support from the Swedish Alzheimer Foundation (Alzheimerfonden), Stiftelsen för Gamla Tjänarinnor, Gun and Bertil Stohnes Foundation, and the Swedish Research Council (2017-01874). Y Yu acknowledges support from the China Scholarship Council (201600160089) and Thomas Olausson. DC Jans acknowledges financial support for infrastructure development from the Swedish Foundation for Strategic Research (RIF14-0091). B Winblad acknowledges financial support from Margaretha af Ugglas' foundation and the Swedish Brain Foundation. Part of the study was performed at the Live Cell Imaging unit/Nikon Center of Excellence, Department of Biosciences and Nutrition, Karolinska Institutet, Huddinge, Sweden, supported by grants from the Knut and Alice Wallenberg Foundation, the Swedish Research Council, the Centre for Innovative Medicine, and the Jonasson donation to the School of Technology and Health, Royal Institute of Technology, Sweden. We thank Henrik Biverstål for kindly providing purified recombinant human A 42 .

\section{Author Contributions}

Y Yu: data curation, software, formal analysis, investigation, visualization, methodology, and writing-original draft, review, and editing.

DC Jans: software, formal analysis, investigation, visualization, methodology, and writing-original draft, review, and editing.

B Winblad: supervision and funding acquisition.

LO Tjernberg: conceptualization, resources, data curation, formal analysis, supervision, funding acquisition, validation, investigation, methodology, project administration, and writing-original draft, review, and editing.

S Schedin-Weiss: conceptualization, data curation, formal analysis, supervision, validation, investigation, visualization, methodology, project administration, and writing-original draft, review, and editing.

\section{Conflict of Interest Statement}

The authors declare that they have no conflict of interest.

\section{References}

1. Raskin J, Cummings J, Hardy J, Schuh K, Dean RA (2015) Neurobiology of Alzheimer's disease: Integrated molecular, physiological, anatomical, biomarker, and cognitive dimensions. Curr Alzheimer Res 12: 712-712. doi:10.2174/1567205012666150701103107
2. Jack CR Jr, Holtzman, DM (2013) Biomarker modeling of Alzheimer's disease. Neuron 80: 1347-1358. doi:10.1016/j.neuron.2013.12.003

3. Haass C, Selkoe DJ (2007) Soluble protein oligomers in neurodegeneration: Lessons from the Alzheimer's amyloid betapeptide. Nat Rev Mol Cell Biol 8: 101-112. doi:10.1038/nrm2101

4. Wilcox KC, Lacor PN, Pitt J, Klein WL (2011) Abeta oligomer-induced synapse degeneration in Alzheimer's disease. Cell Mol Neurobiol 31: 939-948. doi:10.1007/s10571-011-9691-4

5. De Strooper B, Karran E (2016) The cellular phase of Alzheimer's disease. Cell 164: 603-615. doi:10.1016/j.cell.2015.12.056.

6. Hashimoto M, Bogdanovic N, Nakagawa H, Volkmann I, Aoki M, Winblad B, Sakai J, Tjernberg LO (2012) Analysis of microdissected neurons by 180 mass spectrometry reveals altered protein expression in Alzheimer's disease. J Cell Mol Med 16: 1686-1700. doi:10.1111/j.15824934.2011.01441.x

7. Takahashi RH, Milner TA, Li F, Nam EE, Edgar MA, Yamaguchi H, Beal MF, Xu H, Greengard P, Gouras GK (2002) Intraneuronal Alzheimer abeta42 accumulates in multivesicular bodies and is associated with synaptic pathology. Am J Pathol 161: 1869-1879. doi:10.1016/s0002-9440(10)64463-x

8. Nilsson $\mathrm{P}$, Loganathan $\mathrm{K}$, Sekiguchi M, Matsuba Y, Hui K, Tsubuki S, Tanaka M, Iwata N, Saito T, Saido TC (2013) A $\beta$ secretion and plaque formation depend on autophagy. Cell Rep 5: 61-69. doi:10.1016/j. celrep.2013.08.042

9. Spires-Jones TL, Hyman BT (2014) The intersection of amyloid beta and tau at synapses in Alzheimer's disease. Neuron 82: 756-771. doi:10.1016/j. neuron.2014.05.004

10. Steiner H, Fluhrer R, Haass C (2008) Intramembrane proteolysis by gamma-secretase. J Biol Chem 283: 29627-29631. doi:10.1074/jbc. r800010200

11. Willem M, Lammich S, Haass C (2009) Function, regulation and therapeutic properties of beta-secretase (BACE1). Semin Cell Dev Biol 20 175-182. doi:10.1016/j.semcdb.2009.01.003

12. Jan A, Hartley DM, Lashuel HA (2010) Preparation and characterization of toxic Abeta aggregates for structural and functional studies in Alzheimer's disease research. Nat Protoc 5: 1186-1209. doi:10.1038/ nprot.2010.72

13. Welander H, Franberg J, Graff C, Sundstrom E, Winblad B, Tjernberg LO (2009) Abeta 43 is more frequent than Abeta 40 in amyloid plaque cores from Alzheimer disease brains. J Neurochem 110: 697-706. doi:10.1111/ j.1471-4159.2009.06170.x

14. Kamal A, Almenar-Queralt A, LeBlanc JF, Roberts EA, Goldstein LS (2001) Kinesin-mediated axonal transport of a membrane compartment containing beta-secretase and presenilin-1 requires APPAbeta43 is more frequent than Abeta 40 in amyloid plaque cores from Alzheimer disease brains. Nature 414: 643-648. doi:10.1038/414643a

15. Koo EH, Sisodia SS, Archer DR, Martin LJ, Weidemann A, Beyreuther K, Fischer P, Masters CL, Price DL (1990) Precursor of amyloid protein in Alzheimer disease undergoes fast anterograde axonal transport. Proc Natl Acad Sci USA 87: 1561-1565. doi:10.1073/pnas.87.4.1561

16. Gouras GK, Tampellini D, Takahashi RH, Capetillo-Zarate E (2010) Intraneuronal beta-amyloid accumulation and synapse pathology in Alzheimer's disease. Acta Neuropathol 119: 523-541. doi:10.1007/s00401010-0679-9

17. Lundgren JL, Ahmed S, Schedin-Weiss S, Gouras GK, Winblad B, Tjernberg LO, Frykman S (2015) ADAM10 and BACE1 are localized to synaptic vesicles. J Neurochem 135: 606-615. doi:10.1111/jnc.13287

18. Schedin-Weiss S, Caesar I, Winblad B, Blom H, Tjernberg LO (2016) Superresolution microscopy reveals gamma-secretase at both sides of the neuronal synapse. Acta Neuropathol Commun 4: 29. doi:10.1186/s40478016-0296-5

19. Lundgren JL, Ahmed S, Winblad B, Gouras GK, Tjernberg LO, Frykman S (2014) Activity-independent release of the amyloid beta-peptide from 
rat brain nerve terminals. Neurosci Lett 566: 125-130. doi:10.1016/j. neulet.2014.02.050

20. Aoki M, Volkmann I, Tjernberg LO, Winblad B, Bogdanovic N (2008) Amyloid beta-peptide levels in laser capture microdissected cornu ammonis 1 pyramidal neurons of Alzheimer's brain. Neuroreport 19: 1085-1089. doi:10.1097/wnr.0b013e328302c858

21. Hashimoto M, Bogdanovic N, Volkmann I, Aoki M, Winblad B, Tjernberg LO (2010) Analysis of microdissected human neurons by a sensitive ELISA reveals a correlation between elevated intracellular concentrations of Abeta42 and Alzheimer's disease neuropathology. Acta Neuropathol 119: 543-554. doi:10.1007/s00401-010-0661-6

22. Scheff SW, Price DA (2003) Synaptic pathology in Alzheimer's disease: A review of ultrastructural studies. Neurobiol Aging 24: 1029-1046. doi:10.1016/j.neurobiolaging.2003.08.002

23. Coleman PD, Yao PJ (2003) Synaptic slaughter in Alzheimer's disease. Neurobiol Aging 24: 1023-1027. doi:10.1016/j.neurobiolaging.2003.09.001

24. Dani A, Huang B, Bergan J, Dulac C, Zhuang X (2010) Superresolution imaging of chemical synapses in the brain. Neuron 68: 843-856. doi:10.1016/j.neuron.2010.11.021

25. Naslund I, Haroutunian V, Mohs R, Davis KL, Davies P, Greengard P, Buxbaum JD (2000) Correlation between elevated levels of amyloid beta-peptide in the brain and cognitive decline. JAMA 283: 1571-1577. doi:10.1001/jama.283.12.1571

26. Philipson O, Lord A, Lalowski M, Soliymani R, Baumann M, Thyberg J, Bogdanovic N, Olofsson T, Tjernberg LO, Ingelsson M, et al (2012) The arctic amyloid-beta precursor protein (AbetaPP) mutation results in distinct plaques and accumulation of $\mathrm{N}$ - and C-truncated Abeta. Neurobiol Aging 33: 1010.e1-1010.e13. doi:10.1016/j.neurobiolaging. 2011.10.022

27. Schedin-Weiss $S$, Inoue $M$, Hromadkova L, Teranishi Y, Yamamoto NG, Wiehager B, Bogdanovic N, Winblad B, Sandebring-Matton A, Frykman S, et al (2017) Monoamine oxidase B is elevated in Alzheimer disease neurons, is associated with gamma-secretase and regulates neuronal amyloid beta-peptide levels. Alzheimers Res Ther 9: 57. doi:10.1186/ s13195-017-0279-1

28. Imig C, Min SW, Krinner S, Arancillo M, Rosenmund C, Sudhof TC, Rhee J, Brose N, Cooper BH (2014) The morphological and molecular nature of synaptic vesicle priming at presynaptic active zones. Neuron 84: 416-431. doi:10.1016/j.neuron.2014.10.009
29. Yu WH, Cuervo AM, Kumar A, Peterhoff CM, Schmidt SD, Lee JH, Mohan PS, Mercken M, Farmery MR, Tjernberg LO, et al (2005) Macroautophagy: A novel Beta-amyloid peptide-generating pathway activated in Alzheimer's disease. J Cell Biol 171: 87-98. doi:10.1083/jcb.200505082

30. Sahl SJ, Hell SW, Jakobs S (2017) Fluorescence nanoscopy in cell biology. Nat Rev Mol Cell Biol 18: 685-701. doi:10.1038/nrm.2017.71

31. Rust MJ, Bates $M$, Zhuang $X$ (2006) Sub-diffraction-limit imaging by stochastic optical reconstruction microscopy (STORM). Nat Methods 3 : 793-795. doi:10.1038/nmeth929

32. Heilemann $M$, van de Linde $S$, Schuttpelz $M$, Kasper R, Seefeldt $B$, Mukherjee A, Tinnefeld P, Sauer M (2008) Subdiffraction-resolution fluorescence imaging with conventional fluorescent probes. Angewandte Chemie 47: 6172-6176. doi:10.1002/anie.200802376

33. Folling J, Bossi M, Bock H, Medda R, Wurm CA, Hein B, Jakobs S, Eggeling C, Hell SW (2008) Fluorescence nanoscopy by ground-state depletion and single-molecule return. Nat Methods 5: 943-945. doi:10.1038/nmeth.1257

34. Fath T, Ke YD, Gunning P, Gotz J, Ittner LM (2009) Primary support cultures of hippocampal and substantia nigra neurons. Nat Protoc 4: 78-85. doi:10.1038/nprot.2008.199

35. Schedin-Weiss $\mathrm{S}$, Inoue M, Teranishi Y, Yamamoto NG, Karlstrom $\mathrm{H}$, Winblad B, Tjernberg LO (2013) Visualizing active enzyme complexes using a photoreactive inhibitor for proximity ligation: Application on gamma-secretase. PLoS One 8: e63962. doi:10.1371/journal.pone.0063962

36. Dempsey GT, Vaughan JC, Chen KH, Bates M, Zhuang X (2011) Evaluation of fluorophores for optimal performance in localizationbased super-resolution imaging. Nat Methods 8: 1027-1036. doi:10.1038/nmeth.1768

37. Tokunaga M, Imamoto N, Sakata-Sogawa K (2008) Highly inclined thin illumination enables clear single-molecule imaging in cells. Nat Methods 5: 159-161. doi:10.1038/nmeth1171

38. Bernhem K, Brismar H (2018) SMLocalizer, a GPU accelerated Image) plugin for single molecule localization microscopy. Bioinformatics 34: 137-138. doi:10.1093/bioinformatics/btx553

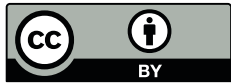

License: This article is available under a Creative Commons License (Attribution 4.0 International, as described at https://creativecommons.org/ licenses/by/4.0/). 